\title{
COMMUTATORS AND ABSOLUTELY CONTINUOUS OPERATORS $\left({ }^{1}\right)$
}

\author{
BY \\ C. R. PUTNAM
}

1. Introduction. In this paper all operators are bounded linear transformations on a Hilbert space consisting of elements $x$. By the (first) commutator $C$ of two operators $A$ and $B$ is meant the difference

$$
C=A B-B A \quad\left(=B^{(1)}\right) .
$$

Similarly one can define higher order commutators $B^{(n)}$ by

$$
B^{(n)}=A B^{(n-1)}-B^{(n-1)} A \quad(n=1,2, \cdots),
$$

where $B^{(0)}=B$. (The commutation operation corresponds to that of differentiation; cf. [3, p. 192].)

By $W=W_{C}$ will be meant the closure of the set of values $(C x, x)$ where $x$ is of length 1 . As in [8], a complex number $z$ will be said to belong to the interior of the convex set $W$ if $z$ is in $W$ and if one of the following conditions holds: If $W$ is two-dimensional, $z$ is not on the boundary of $W$; if $W$ is a line segment, $z$ is not an end-point; or, finally, $W$ consists of the single value $z$ alone.

In [6], it was shown that if $A$ (or $B$ ) is normal or even semi-normal, so that $A A^{*}-A^{*} A$ is semi-definite, then 0 is in the set $W$. (If $A$ is arbitrary, 0 need not even belong to $W$; [2].) In [8], it was supposed that $A$ is actually normal, with a spectral resolution

$$
A=\int z d K
$$

and the problem of determining sufficient conditions guaranteeing that 0 be in the interior of $W$ was considered.

The present paper will depend upon the methods of [8] and upon certain consequences and extensions of results obtained there. The paper will be divided in to two parts; Part I will consist of general theorems on commutators $C$ and the associated sets $W_{C}$, while Part II will be devoted to applications of some of these results, in particular to Toeplit\%, Hankel, and Jacobi matrices.

\section{PakT I}

2. It will be convenient to recall here for later use a result proved in [8],

Received by the editors October 18, 1956.

(1) This research was supported in part by the Inited States Air Force under Contraci No. AF 18 (603)-139, monitored by the . IF Office of Scientific Research of the Air Research and Development Command. 
namely, if $A$ is self-adjoint or unitary and if (for a fixed $B$ ) $C$ is defined by (1) and is such that $H=C+C^{*} \geqq 0$, then

$$
\left\|H^{1 / 2} \int_{S} d K x\right\|^{2} \leqq 4\|B\|\|x\|^{2} \text { meas } S
$$

for any measurable set $S$, the measure being the ordinary one-dimensional Lebesgue measure. From this result it was proved loc. cit. that if $A$ is selfadjoint or unitary and if 0 is not in the interior of $W_{C}$, then

$$
\int_{z} d K<I
$$

for every set $Z$ of one-dimensional measure 0 .

It is to be noted that the relation $\int_{Z} d K=I$ for some zero set $Z$ is not incompatible with the existence of a purely continuous spectrum (no point spectrum) consisting of, say, a single interval. Needless to say, the closure of such a zero set would necessarily contain the aforementioned interval.

In the present paper there will be proved results similar to the above but for second and third commutators,

$$
D\left(=B^{(2)}\right)=A C-C A \text { and } E\left(=B^{(3)}\right)=A D-D A,
$$

respectively. In fact, the following two theorems will be proved:

THEOREM 1. If $A$ is normal with the spectral resolution (3) and if 0 is not in the interior of $W_{D}$, where $D$ is defined by (6), then (5) holds for every set $Z$ of twodimensional measure 0.

Since the spectrum of a self-adjoint or unitary operator is always of twodimensional measure 0 , one obtains the following

Corollary of Theorem 1. If $A$ is self-adjoint or unitary, then 0 is in the interior of $W_{D}$.

Theorem 2. If $A$ is normal, then 0 is always in the interior of $W_{E}$, where $E$ is defined by (6).

Since any $n$th order commutator $B^{(n)}$ for $n=4,5, \cdots$ is also a third commutator (of $B^{(n-3)}$ in fact) it follows of course from Theorem 2 that, when $A$ is normal, 0 is always in the interior of $W_{B(n)}$ for $n=3,4,5, \cdots$.

3. The assertion (5) of Theorem 1 can be improved to

$$
\int_{Z} d K=0
$$

if certain additional restrictions are imposed. In fact there will be proved the following two theorems:

THEOREM 3. Let $A$ be self-adjoint or unitary and suppose that 0 is not in 
the interior of $W_{C}$. In addition, suppose that there exists a line $l$ in the complex plane passing through the origin, lying entirely on one side of $W_{C}$, and such that no number $(C x, x)$, for $\|x\|=1$, lies on $l$. Then (7) holds for every set $Z$ of onedimensional measure 0.

Of course, since $A$ is normal, 0 is in the set $W_{C}$ [6], so that there exist numbers $(C x, x)$ with $\|x\|=1$ clustering at 0 ; it is required however that these numbers do not lie on $l$. A similar remark applies to the set $W_{D}$ in the theorem below.

TheOREM 4. Let $A$ be normal and suppose that 0 is not in the interior of $W_{D}$. Suppose that there exists a line corresponding to the set $W_{D}$ as $l$ does to $W_{C}$ above. Then (7) holds for every set $Z$ of two-dimensional measure 0.

Theorem 3 can be regarded as furnishing a sufficient condition in order that a self-adjoint or a unitary operator be absolutely continuous. Here the last term is borrowed from the terminology occurring in the treatment of real functions. What is meant is the following: A self-adjoint or unitary operator with a spectral resolution (3) will be called absolutely continuous if $\int_{z} d K=0$ for every set $Z$ of one-dimensional measure zero. For a self-adjoint operator $A=\int \lambda d E(\lambda)$, absolute continuity is thus equivalent to the requirement that $\|E(\lambda) x\|$ be an absoutely continuous function of the real variable $\lambda$ for every fixed element $x$ of Hilbert space; a similar remark of course holds if $A$ is unitary.

Similarly, Theorem 4 can be regarded as furnishing a sufficient condition for absolute continuity of a general normal operator. It should be emphasized however that the measure here is two-dimensional.

It is to be noted that a necessary, but not sufficient, condition in order that an operator be absolutely continuous is that it possesses no point spectrum.

The proofs of Theorems $1-4$ will be given in $\S \S 4-7$ below.

4. Proof of Theorem 1. The proof is similar to that of the lemma and theorem in [8] and will be outlined here. Multiplication of both sides of the Equation (1) by $\Delta K\left(=\int_{\Delta} d K, \Delta\right.$ any measurable set) on the right and on the left yields

$$
\Delta K C \Delta K=\int_{\Delta}\left(z-z_{0}\right) d K B \Delta K-\Delta K B \int_{\Delta}\left(z-z_{0}\right) d K,
$$

where $z_{0}$ is an arbitrary constant. Next, choose $\theta$ so that the set $W_{D} e^{i \theta}$ belonging to

$$
D_{\theta}=A C_{\theta}-C_{\theta} A \text {, where } C_{\theta}=C e^{i \theta} \text { and } D_{\theta}=D e^{i \theta},
$$

lies in the half-plane $R(z) \geqq 0$. Thus $J_{\theta}=D_{\theta}+D_{0}^{*} \geqq 0$. Multiplication of both sides of (9) on the right and left by $\Delta K$ yields $\Delta K D_{\theta} \Delta K=\int_{\Delta}\left(z-z_{0}\right) d K C_{\theta} \Delta K$ $-\Delta K C_{\theta} \int_{\Delta}\left(z-z_{0}\right) d K$. It now follows from (8) that $\left\|\Delta K D_{\theta} \Delta K x\right\| \leqq 2 d\|\Delta K C \Delta K x\|$ $\leqq 4 d^{2}\|B\|\|\Delta K x\|$, where $d$ is the diameter of the set $\Delta$. Since a similar relation 
holds also for $D_{\theta}^{*}$, one readily obtains the inequality $\left(\Delta K x, J_{\theta} \Delta K x\right)$ $\leqq 8\|B\|\|\Delta K x\|^{2} d^{2}$, hence $\left\|J_{\theta}^{1 / 2} \Delta K x\right\| \leqq 8^{1 / 2}\|B\|^{1 / 2}\|\Delta K x\| d$. If now $\left\{\Delta_{1}, \Delta_{2}, \cdots\right\}$ is a covering by pairwise disjoint sets of a measurable set $S$ and if $d_{n}$ is the diameter of $\Delta_{n}$, one obtains the inequality

$$
\left\|J_{\theta}^{1 / 2} \int_{S} d K x\right\| \leqq 8^{1 / 2}\|B\|^{1 / 2}\|x\|\left(\sum_{n} d_{n}^{2}\right)^{1 / 2} ;
$$

cf. [8]. If $S=Z$ is a set of two-dimensional measure 0 it is clear that $\sum d_{n}^{2}$ can be made arbitrarily small and so one obtains

$$
J_{\theta}^{1 / 2} \int_{z} d K=0
$$

If however $\int_{z} d K=I$ were true for some zero set $Z$, then $J_{\theta}^{1 / 2}$, hence $J_{\theta}$, would be the zero operator and it would follow, as in [8], that 0 lies in the interior of $W_{D}$, a contradiction. 'This completes the proof of Theorem 1.

5. Proof of Theorem 2. An examination of the proof of Theorem 1 shows that in the present case the inequality (10) is replaced by $\left\|L_{\theta}^{1 / 2} \int_{S} d K x\right\|$ $\leqq 4\|B\|{ }^{1 / 2}\|x\|\left(\sum_{n} d_{n}^{3}\right)^{1 / 2}$, where $L_{\theta}=E_{\theta}+E_{\theta}^{*}\left(E_{\theta}=E e^{i \theta}\right)$ and the sets $\Delta_{n}$ and numbers $d_{n}$ have the same significance as before. If the set $S$ is chosen so that $\int_{S} d K=I$, then $\left\|L_{\theta}^{1 / 2}\right\| \leqq\left(\sum d_{n}^{3}\right)^{1 / 2}$. Since this sum can be made arbitrarily small, $L_{0}=0$ and, as before, a contradiction is obtained. This completes the proof of Theorem 2.

6. Proof of Theorem 3. The proof of Theorem 3 is an easy consequence of (4). In fact, since 0 is not in the interior of $W_{C}$ there exists an angle $\theta$ such that $H_{\theta}=C_{\theta}+C_{\theta^{*}} \geqq 0$. Moreover, in view of the assumption of Theorem 3 , it follows that $\left(H_{\theta} x, x\right)>0$ for every $x \neq 0$. Thus, 0 is not in the point spectrum of $H_{\theta}$. On the other hand, if $Z$ is any set of one-dimensional measure 0 , relation (4) implies $H_{\theta}^{1 / 2} \int_{Z} d K=0\left(=\int_{Z} d K H_{\theta}^{1 / 2}\right)$ is valid; cf. [8]. Consequently, relation (7) follows and the proof of Theorem 3 is now complete.

7. Proof of Theorem 4. There exists some angle $\theta$ for which (11) holds. The assumption of the theorem implies, as in the preceding proof, that 0 is not in the point spectrum of $J_{\theta}^{1 / 2}$. Relation (7) then follows from (11) and the proof of Theorem 4 is complete.

8. The proof of Theorem 3 makes clear the following assertion, which will be stated as a theorem and will be of later use:

THEOREM 5. Let $A$ be self-adjoint or unitary. Suppose that there exist operators $B_{1}, B_{2}, \cdots$ such that $H_{n}=C_{n}+C_{n}{ }^{*}$ is semi-definite, where $C_{n}=A B_{n}$ $-B_{n} A$, and such that $\sum \Re\left(I I_{n}^{1 / 2}\right)$ is dense in the Iilbert space. Then $A$ is absolutely continuous, so that (7) holds, for every set $Z$ of one-dimensional measure 0 .

\section{PART II}

9. Let $c_{n}(n=0, \pm 1, \pm 2, \cdots)$ be a sequence of complex numbers satisfying 


$$
c_{-n}=\bar{c}_{n}, \quad \sum_{n=-\infty}^{\infty}\left|c_{n}\right|^{2}<\infty
$$

and let $T$ denote the Toeplitz matrix defined by $T=\left(c_{k-j}\right)$, for $j, k=0,1$, $2, \cdots$. For references, see $[4 ; 5 ; 10]$. A necessary and sufficient condition for the boundedness of $T$ is that the function $f(\theta)$ defined by the Fourier series $f(\theta) \sim \sum_{-\infty}^{\infty} c_{n} e^{i n \theta}$ be bounded almost everywhere (Toeplitz; cf. [4, p. 360]). It was proved by Hartman and Wintner $[5$, p. 868] that, if $T$ is bounded, its spectrum is a closed interval $[m, M]$, where $m$ and $M$ denote the bounds of $f(\theta)$; furthermore, if $T$ is not a multiple of the unit matrix, its point spectrum is empty.

Other results, concerning absolute continuity and the spectra of Toeplitz matrices, will be obtained in this paper.

First, define a matrix $A=\left(a_{j k}\right)$ as follows:

$$
a_{j k}=c_{k-j} \text { for } k-j \geqq 1 \text { and } a_{j k}=0 \text { otherwise. }
$$

Thus the main diagonal of $A$, and those below it, consist entirely of zeros. It is clear that the general Toeplitz matrix $T$ is given by

$$
T=A+A^{*}+c_{0} I .
$$

As was mentioned at the beginning of this paper, all operators are supposed bounded. As was pointed out in [5, p. 880], it follows from Toeplitz's results on self-adjoint operators that the above mentioned necessary and sufficient condition for the boundedness of $T$ holds also for operators for which the second, but not necessarily the first, condition of (12) is satisfied. In particular, the above mentioned $A$ is bounded if and only if $f(\theta)$ (of class $L^{2}[0,2 \pi]$ ) defined by $f(\theta) \sim \sum_{1}^{\infty} c_{n} e^{i n \theta}$ is bounded (almost everywhere). It is to be noted that the boundedness of $A$ implies, but is not implied by, the boundedness of $T$.

Direct calculation shows that

$$
\left\|A^{*} x\right\|^{2}-\|A x\|^{2}=\sum_{n=2}^{\infty}\left|\sum_{k=1}^{n-1} \bar{c}_{n-k} x_{k}\right|^{2}-\sum_{n=1}^{\infty}\left|\sum_{k=n+1}^{\infty} c_{k-n} x_{k}\right|^{2} .
$$

On the other hand, the right side of this last equality is equal to

$$
\sum_{m=0}^{\infty}\left|\sum_{j=1}^{\infty} c_{j+m} x_{j}\right|^{2} \geqq 0
$$

A proof of this claim follows, for instance, from a comparison of the coefficients of the terms $x_{r} \bar{x}_{s}$. In fact, if the $x_{k}$ and the $\bar{x}_{k}$ are regarded as two sets of independent variables, it is seen that the coefficient of $x_{r} \bar{x}_{s}(r \leqq s)$ in (15) is $\sum_{n=s}^{\infty} \bar{c}_{n-r+1} c_{n-s+1}-\sum_{n=1}^{r-1} c_{r-n} \bar{c}_{s-n}$, that is $\sum_{m=0}^{\infty} c_{r+m} \bar{c}_{s+m}$, the coefficient of $x_{r} \bar{x}_{8}$ in (16).

10. It is of interest to note here that the matrix $A$ defined by (13) is semi-normal, thus 


$$
C=A A^{*}-A^{*} A \geqq 0 .
$$

The following result for arbitrary semi-normal operators will be proved:

Theorem 6. Let $A$ be an arbitrary semi-normal operator and let $C=A A^{*}$ $-A^{*} A$. Then either $C=0$ (that is, $A$ is normal) or 0 is in the essential spectrum of $C$.

A point $\mu$ is said to be in the essential spectrum of $C$ if $\mu$ is either an eigenvalue of infinite multiplicity or a cluster point of points in the spectrum of $C$ (or both). Of course, if $C=0$ and is not a finite matrix, then 0 is in the essential spectrum. Somewhat more than Theorem 6 is contained in the following:

Theorem 7. Let $A$ be normal and suppose that $C$ of (1) satisfies $H=C+C^{*}$ $\geqq 0$. Then either $H=0$ or 0 is in the essential spectrum of $H$.

That Theorem 6 is a consequence of Theorem 7 is clear if it is noted that $C$ of (17) is self-adjoint and that $A$ can be replaced by the self-adjoint, hence normal, operator $A+A^{*}$.

11. Proof of Theorem 7. If $A$ has a pure point spectrum (in particular, if $A$ is finite) it follows from Corollary 1 of [8] that 0 is in the interior of $W_{C}$, hence, since $H \geqq 0, H=0$.

Otherwise, let $\mu$ denote a cluster point of points in the continuous spectrum of $A$. Then choose an element $x$ and sets $\Delta_{n}$, with diameters $d_{n}$, such that the $\Delta_{n}$ tend to the point $\mu$, and the elements $y_{n}=\Delta_{n} K x /\left\|\Delta_{n} K x\right\|$, of length 1 , tend weakly to zero when $n \rightarrow \infty$. As was shown in [8] (cf. (4) of the present paper), $\left\|H^{1 / 2} y_{n}\right\| \leqq$ const. $d_{n}^{1 / 2} \rightarrow 0$ as $n \rightarrow \infty$. Thus 0 is in the essential spectrum of $C$ and the proof of Theorem 7 is now complete.

12. Next there will be proved the

Theоrem 8. Let $T$ be any Toeplitz matrix (bounded, self-adjoint, and such that the corresponding matrix $A$ defined by (13) is also bounded) with the spectral resolution

$$
T=\int \lambda d E(\lambda)
$$

Then, unless $T$ is a multiple of the unit matrix $I$,

$$
\int_{z} d E<I
$$

for every set $Z$ of one-dimensional measure 0 .

Proof of Theorem 8 . It is seen that $C=A A^{*}-A^{*} A \geqq 0$, where $A$ is defined by (13). Hence, by Corollary 3 of [8], either (i) $C=0$ or (ii) relation (19) holds. In fact, in the corollary mentioned, it is clear that the assertion remains true if $A+A^{*}$ there is replaced by $A+A^{*}+\lambda I$ for any complex number $\lambda$. 
It is to be noted that in view of (14), $C=T A^{*}-A^{*} T(\geqq 0)$.

If case (i) holds, then by (16),

$$
\sum_{j=1}^{\infty} c_{j+m} x_{j}=0 \quad \text { for } m=0,1,2, \cdots\left(\text { whenever } \sum\left|x_{k}\right|^{2}<\infty\right),
$$

and so $c_{k}=0$ for $k=1,2,3, \cdots$. Thus $T$ is a multiple of $I$ and the proof of Theorem 6 is now complete.

Remark. As was mentioned earlier, it is known [5] that if $T$ is not a multiple of the unit matrix, then its spectrum is an interval and its point spectrum is empty. This fact alone does not seem to imply (19) however; cf. the remark following formula line (5).

It is natural to ask whether a Toeplitz matrix (not a multiple of $I$ ) is absolutely continuous. This question will remain undecided in the general case; however, it will be shown that certain Toeplitz matrices do possess this property.

13. Let $T_{n}(c)$ denote the Toeplitz matrix belonging to the sequence $\left\{c_{k}\right\}$ in which $c_{n}=c, c_{-n}=\bar{c}$ and all other $c_{k}=0$. In particular, $T_{1}(1)$ is the Jacobi matrix belonging to the (real) quadratic form $\sum 2 x_{n} x_{n+1}$. There will be proved the following

Theorem 9. Every Toeplitz matrix

$$
T_{n}(c)=\int \lambda d E_{n}(\lambda), \quad \text { where } n=1,2,3, \cdots .
$$

for which

$$
c \neq 0 \text { and is real or purely imaginary, }
$$

is absolutely continuous, that is

$$
\int_{Z} d E_{n}=0
$$

for every set $Z$ of one-dimensional measure 0 . (See $\$ 1$ of the Appendix.)

The restriction (22) amounts to restricting $T_{n}(c)$ to be a multiple of $T_{n}(1)$ or $T_{n}(i)$. Whether the theorem remains true for arbitrary $c$ will remain undecided.

14. Proof of Theorem 9. For a fixed $n$ consider the matrix $T_{n}(c)$. For $m=1,2, \cdots$ construct a (bounded) matrix $B_{n m}$ as follows: The first $m n$ rows of $B_{n m}$ consist entirely of zeros. For $k=0,1,2, \cdots, m-1$, and beginning with the element in the $(n(m+k)+1, n(m-1-k)+1)$ position and extending in a southeast direction, construct a diagonal each element of which is a $c$ or a $\bar{c}$ according as $k$ is even or odd. All other clements are zeros. For instance, for $n=1, m=3$, one obtains the matrix $B_{13}$ defined by 


$$
B_{13}=\left(\begin{array}{ccccccccccc}
0 & 0 & 0 & 0 & 0 & 0 & . & . & . & . & . \\
0 & 0 & 0 & 0 & 0 & 0 & . & . & . & . & . \\
0 & 0 & 0 & 0 & 0 & 0 & . & . & . & . & . \\
0 & 0 & c & 0 & 0 & 0 & . & . & . & . & . \\
0 & \bar{c} & 0 & c & 0 & 0 & . & . & . & . & . \\
c & 0 & \bar{c} & 0 & c & 0 & . & . & . & . & . \\
0 & c & 0 & \bar{c} & 0 & c & . & . & . & . & . \\
. & . & . & . & . & . & . & . & . & . & .
\end{array}\right)
$$

It is to be noted that, in view of $(22), c^{2}=\bar{c}^{2}(\neq 0)$. It can be verified directly that $C_{n m}=T_{n}(c) B_{n m}-B_{n m} T_{n}(c)$ is a diagonal matrix all of whose elements are zero except for a string of $n$ elements from the $n(m-1)+1$ diagonal element through the $m n$ diagonal element each of which is $c^{2}$.

Consequently, each matrix $C_{n m}$ is semi-definite and, moreover, for a fixed $m$, the range of $C_{n m}^{1 / 2}$, that is $\Re\left(C_{n m}^{1 / 2}\right)$, is the space of vectors $x$ all of whose components are zero except those from the $n(m-1)+1$ through the $m n$ element. Clearly (for $n$ fixed) the spaces $\Re\left(C_{n m}^{1 / 2}\right)$ for $m=1,2,3, \cdots$ are orthogonal and moreover their sum is the entire Hilbert space. Relation (19) is now a consequence of Theorem 5 and the proof of Theorem 9 is complete.

Remark. For a fixed $m$, choose real numbers $\alpha_{n m} \neq 0$ such that $\alpha_{n m} C_{n m}$ $=T_{n}\left(\alpha_{n m} B_{n m}\right)-\left(\alpha_{n m} B_{n m}\right) T_{n} \geqq 0$ and such that $\sum_{m=1}^{\infty}\left|\alpha_{n m}\right|\left\|B_{n m}\right\|<\infty$. It is clear that $B=\sum_{m} \alpha_{n m} B_{n m}$ is bounded and that $C=\sum_{m} \alpha_{n m} C_{n m} \geqq 0$. In fact, $C$ is a diagonal matrix with diagonal elements all positive. Thus 0 is not in the point spectrum of $C$. Consequently, Theorem 9 would now follow from Theorem 3.

Moreover, the above furnishes an example of an $I I(=2 C)$ in Theorem 7 in which 0 is in the essential spectrum but is not in the point spectrum.

15. Henceforth, only $T_{n}(c)$ for $c$ real will be considered. Let $T_{n}=T_{n}(1)$ for $n=1,2,3, \cdots$; then, of course, $T_{n}(c)=c T_{n}$. Let $I I=\left(c_{k+j-1}\right)$ denote the Hankel matrix associated with the elements $c_{n}$ considered in the beginning of $\S 9$. (For results on such matrices, see [4].) For a fixed $n$, let $H_{n}(c)$ denote the Hankel matrix belonging to the sequence $\left\{c_{k}\right\}$ in which $c_{n}=c$ and all other $c_{k}=0$; in particular, if $c$ is real, $H_{n}(c)=c H_{n}(1)=c H_{n}$ is self-adjoint. The following will be proved:

Theorem 10. For every $n=1,2,3, \cdots$, the Toeplitz matrix $T_{n}$ can be expressed as

$$
T_{n}=p_{n}\left(T_{1}\right)+H_{n-1},
$$

where $p_{n}\left(T_{1}\right)=\sum_{k=0}^{n} a_{k} T_{1}^{k}$ denotes a polynomial of degree $n$ in $T_{1}$ with real coefficients $a_{k}$, and $a_{n}=1$. Moreover, the polynomial contains only odd, or only even, powers of $T_{1}$ according as $n$ is odd or even. (See $\$ 2$ of the Appendix.) 
Proof of Theorem 10. The proof, which will be outlined below, depends upon the easily verified relations

$$
\begin{gathered}
T_{1} T_{n}-T_{1} H_{n-1}=T_{n+1}+T_{n-1}-H_{n-2}-H_{n} \quad(n=2,3,4, \cdots), \\
T_{1}^{2}=T_{2}-H_{1}+2 I, \quad T_{1}^{3}=T_{3}-H_{2}+3 T_{1} .
\end{gathered}
$$

In order to apply an induction process, grant that

$$
T_{1}^{k}=T_{k}-H_{k-1}+f_{k}\left(T_{1}\right)
$$

holds for $k=n-1$ and $k=n$ ( $n \geqq 3$, arbitrary), where $f_{k}\left(T_{1}\right)$ denotes a polynomial in $T_{1}$ of degree $k-2$, with leading coefficient $k$, and containing only powers of $T_{1}$ differing from $k-2$ by an even integer. By (26), relation (27) surely holds for $k=n-1, n$ when $n=3$. Multiplication by $T_{1}$ on the left of (27) for $k=n$ yields, in view of (25), $T_{1}^{n+1}=T_{n+1}+T_{n-1}-H_{n-2}-H_{n}+T_{1} f_{n}\left(T_{1}\right)$. Hence by (27) for $k=n-1$, one obtains

$$
T_{1}^{n+1}=T_{n+1}-H_{n}+g_{n+1}\left(T_{1}\right),
$$

where $g_{n+1}\left(T_{1}\right)=T_{1} f_{n}\left(T_{1}\right)-f_{n-1}\left(T_{1}\right)+T_{1}^{n-1}$. Thus $g_{n+1}\left(T_{1}\right)$ is a polynomial of degree $n-1$ with leading coefficient $n+1$. Since relation (28) is simply (27) for $k=n+1$, the induction is now complete. The assertion of Theorem 10 now follows from (27) valid for $k=2,3,4, \cdots$.

Relation (24) shows that the spectrum of each $T_{n}$ is closely related to that of $T_{1}$. Moreover, since $H_{n-1}$ is finite dimensional and hence, in particular, is completely continuous, it follows that the essential spectrum of $T_{n}$ is identical with that of $p_{n}\left(T_{1}\right)$. Moreover, the spectrum of $T_{n}$ is purely continuous and consists of the interval $[-2,2] ;[5$, p. 868]. It will be shown that the following is true:

TheOREM 11. For each $n=1,2, \cdots$ there exists a unitary operator $U_{n}$ such that

$$
T_{n}=U_{n} p_{n}\left(T_{1}\right) U_{n}^{*} \quad\left(=p_{n}\left(U_{n} T_{1} U_{n}^{*}\right)\right),
$$

where $p_{n}\left(T_{1}\right)$ is defined in Theorem 10. (See $\$ 3$ of the Appendix.)

Thus each $T_{n}$ is a polynomial in an operator unitarily equivalent to $T_{1}$. The proof of the theorem will depend upon a theorem of Rosenblum [9, p. 3].

16. Proof of Theorem 11. It was shown in Theorem 9 that each $T_{n}$ is absolutely continuous. (See $\S 4$ of Appendix.) Moreover, since $T_{1}$ in particular is absolutely continuous, it follows that each operator $p_{n}\left(T_{1}\right)$ is also absolutely continuous.

In order to prove this last assertion, note that if $T_{1}=\int \lambda d E_{1}(\lambda)$, then $p_{n}\left(T_{1}\right)=\int p_{n}(\lambda) d E_{1}(\lambda)=\int \lambda d F(\lambda)$, where the last integral is the spectral resolu- 
tion of the self-adjoint operator $p_{n}\left(T_{1}\right)$. Let $Z$ denote an arbitrary set of onedimensional measure 0 . Then

$$
\int_{z} d F(\lambda)=\int_{z^{*}} d E_{1}(\lambda)
$$

where $Z^{*}$ denotes the set of values $\lambda$ for which $p_{n}(\lambda)$ belongs to $Z$. Since $p_{n}(\lambda)$ is a (nonconstant) polynomial the graph of its inverse function $g$ consists of a finite number of (open) monotone, smooth arcs, the ends of which correspond to $\lambda$ values at which $d p_{n}(\lambda) / d \lambda=0$. Thus $g$, or rather each of the single-valued functions corresponding to each of its branches, is an absolutely continuous real-valued function and therefore $Z^{*}$ is a set of one-dimensional measure 0 . Since $T_{1}$ is absolutely continuous, it follows from (30) that $p_{n}\left(T_{1}\right)$ is also.

Since $H_{n-1}$ occurring in (24) is a finite matrix the trace condition of Rosenblum's theorem in $[9$, p. 3$]$, is surely satisfied and the existence of the unitary operator $U_{n}$ of (29) now follows from his result. (Incidentally, Rosenblum requires even a weaker form of absolute continuity in his theorem than actually prevails in the present instance.)

17. It follows from (24) that

$$
\sum_{n=0}^{N} c_{n} T_{n}=P_{N}\left(T_{1}\right)+\sum_{n=1}^{N} c_{n} H_{n-1}
$$

where the $c_{k}$ denote real constants and $P_{N}\left(T_{1}\right)=c_{0} I+\sum_{n=1}^{N} c_{n} p_{n}\left(T_{1}\right)$ is a polynomial of degree $N$ (assuming, for $N \geqq 1$ fixed, that $c_{N} \neq 0$ ). As in the preceding proof, $P_{N}\left(T_{1}\right)$ is absolutely continuous and thus one obtains the result:

THEOREM 12. If $T$ is the self-adjoint Toeplitz matrix associated with the real sequence $\left\{\cdots 0,0, c_{N}, c_{N-1}, \cdots, c_{1}, c_{0}, c_{1}, \cdots, c_{N}, 0,0, \cdots\right\}$, where $c_{N} \neq 0, N \geqq 1$, then there exists an absolutely continuous self-adjoint operator $G$ and a finite-dimensional self-adjoint Hankel matrix $H$ such that

$$
T=G+H \text {. }
$$

Whether $T$ itself is also absolutely continuous will remain undecided. In fact, it will remain undecided whether or not such a simple Toeplitz matrix as $T_{1}+T_{2}$, for instance, is absolutely continuous.

18. If it is assumed that

$$
\text { the series } \sum_{k}\left|c_{k}\right| \text { is convergent, }
$$

it is seen that $\left\|T-\sum_{n=0}^{N} c_{n} T_{n}\right\| \rightarrow 0$ and $\left\|H-\sum_{n=1}^{N} c_{n} H_{n-1}\right\| \rightarrow 0$ as $N \rightarrow \infty$ where $T$ is the (real) Toeplitz matrix belonging to the sequence $\left\{c_{k}\right\}$ and $H$ is a completely continuous Hankel matrix. (That $H$ is completely continuous follows, for instance, from the criterion of $[4$, p. 365].) If, in addition, it is 
assumed that the series of (33) converges rapidly enough to guarantee that $f\left(T_{1}\right)=c_{0} I+\sum_{n=1}^{\infty} c_{n} p_{n}\left(T_{1}\right)$ is a power series in $T_{1}$ (more precisely, that $f(\lambda)$ $=c_{0}+\sum c_{n} p_{n}(\lambda)$ is a power series in $\lambda$ convergent at least for $\left.|\lambda| \leqq\left\|T_{1}\right\|=2\right)$, then a theorem valid for the infinite sequence $\left\{c_{k}\right\}$ and similar to Theorem 12 also holds. ( $H$ of course must now be allowed to be infinite dimensional.)

It should be noted that $f\left(T_{1}\right)$ is a multiple of $I$ only if $c_{k}=0$ for $k=1,2$, $3, \cdots$. In fact, if $c_{k} \neq 0$ for some $k \geqq 1, \sum_{n=0}^{\infty} c_{n} T_{n}$ is not a multiple of $I$ and possesses a purely continuous spectrum ([4] or [5]). Hence, if $f\left(T_{1}\right)$ were a multiple of $I$, then $T=f\left(T_{1}\right)+H$ would have only one point in its essential spectrum, a contradiction.

Clearly that portion of the proof of Theorem 11 relating to the inverse of the polynomial $p_{n}(\lambda)$, now corresponding to the inverse of $f(\lambda)$, is still valid if it is noted that, on any finite interval, $d f(\lambda) / d \lambda=0$ holds for at most a finite number of values.

Lastly, it can be remarked that (33) is surely enough to guarantee that the polynomials $P_{N}(\lambda)=c_{0}+\sum_{k=1}^{n} c_{k} p_{k}(\lambda)$ converge uniformly to a (continuous) function $g(\lambda)$, so that (cf. (31))

$$
T=\int g(\lambda) d E_{1}(\lambda)+H,
$$

where $H$ is completely continuous.

19. In this section there will be considered another connection between Toeplitz and Hankel matrices. Let the numbers $c_{n}$ of (12) be real and suppose that $A$ defined by (13) is bounded. Then $T$ of (14) and also $[4$, p. 365] the Hankel matrix

$$
J=\sum_{n=1}^{\infty} c_{n} H_{n}
$$

is bounded. If $C=A A^{*}-A^{*} A$, relations (15) and (16) become $(C x, x)$ $=|J x|^{2} \geqq 0$ and hence, if 0 is not in the point spectrum of $J,(C x, x)>0$ for every $x \neq 0$. Since $C=T A^{*}-A^{*} T$, Theorem 3 now implies the following

THEOREM 13. Let the numbers $c_{n}$ of (12) be real and let $T$ satisfy the same assumptions as in Theorem 8. If, in addition, 0 is not in the point spectrum of the Hankel matrix $J$ of (35), then the assertion (19) of Theorem 8 can be sharpened to $\int_{Z} d E=0$, for every set $Z$ of one-dimensional measure 0 .

20. This last section will deal with Jacobi matrices. Given a bounded sequence of complex numbers $b_{i}$, define, as in [8], a matrix $A=\left(a_{i j}\right)$ by putting $a_{i, i+1}=b_{i}$ and $a_{i j}=0$ for $j \neq i+1$, so that $D=A+A^{*}=\left(d_{i j}\right)$ is the selfadjoint Jacobi matrix with $d_{i, i+1}=b_{i}, d_{i+1, i}=b_{i}$ and $d_{i j}=0$ otherwise. Then $C=D A^{*}-A^{*} D$ is the diagonal matrix with diagonal elements $\left|b_{1}\right|^{2},\left|b_{2}\right|^{2}$ $-\left|b_{1}\right|^{2},\left|b_{3}\right|^{2}-\left|b_{2}\right|^{2}, \cdots$. It was shown in [8] that if the inequalities 


$$
0<\left|b_{1}\right| \leqq\left|b_{2}\right| \leqq\left|b_{3}\right| \leqq \cdots \quad \text { (<const.) }
$$

hold, then the Jacobi matrix $D=\int \lambda d E(\lambda)$ is such that $\int_{Z} d E<I$ holds for every set $Z$ of one-dimensional measure 0 . If the strict inequalities of (36) prevail, then a refinement of this assertion is contained in the following

THEOREM 14. Suppose that the inequalities

$$
0<\left|b_{1}\right|<\left|b_{2}\right|<\left|b_{3}\right|<\cdots \quad \text { (<const.) }
$$

hold. Then the Jacobi matrix $D$ is absolutely continuous, so that $\int_{Z} d E=0$ for every set $Z$ of one-dimensional measure 0 .

The proof follows immediately from Theorem 3 if it is noted that, in view of $(37)$, the number 0 is not in the point spectrum of the positive semidefinite diagonal matrix $C$.

Suppose, for instance, that the $b_{i}$ are real and positive. Then the matrix $D$ is absolutely continuous in either of the "extreme" instances of (36), namely (37) or

$$
0<b_{1}=b_{2}=b_{3}=\cdots(=b) .
$$

In fact, in case (38), $D=b T_{1}$. It is of interest therefore to inquire whether (36) alone is enough to ensure absolute continuity, even in the case where all $b_{i}$ are real and positive. This question will remain undecided.

\section{Appendix (Added in Proof).}

1. The late Professor Wintner called the author's attention to the references Hilbert [11, p. 155] and Hellinger [12, pp. 148 ff.], wherein are given explicit formulas, in matrix form, for the resolution of the identity belonging to $T_{1}(1)$. The absolute continuity of $T_{1}(1)$ can be immediately inferred. Furthermore, from [12], it is clear that for any integer $n \geqq 1$, the basic Hilbert space $H$ can be expressed as the sum of $n$ pairwise orthogonal spaces $H_{m}$, each of which is invariant under $T_{n}(1)$, and on each of which $T_{n}(1)$ acts like $T_{1}(1)$ on $H$. The absolute continuity of $T_{n}(1)$ can then be deduced from that of $T_{1}(1)$. (Similar results can probably be obtained in this way for $T_{1}(i)$ and $T_{n}(i)$.) The proof of Theorem 9 as given in the present paper involves no explicit formulas for the spectral resolution of $T_{1}(1)$ however.

2. Under the assumptions that the $c_{n}$ satisfy $c_{-n}=c_{n}$ and $\sum_{1}^{\infty} c_{n}^{2}<\infty$, put $T=\left(c_{j-k}\right), H=\left(c_{j+k}\right), F(\theta)=2 \sum_{1}^{\infty} c_{n} \cos n \theta$ and $d \rho_{j k}(\theta)=2 \pi^{-1} \sin j \theta \sin k \theta d \theta$. If $\lambda=2 \cos \theta$, it can be shown from the calculations of $\$ 15$ that $p_{n}(\lambda)$ $=2 \cos n \theta\left(p_{n}(\lambda) / 2\right.$ is the $n$th degree Tschebyscheff polynomial $\left.\lambda / 2\right)$ and that

$$
T=c_{0} I+\left(\int_{0}^{\pi} F(\theta) d \rho_{j k}(\theta)\right)+H .
$$

Actually however a simple and immediate proof of (39) is obtained by direct 
verification. The matrix $\left(d \rho_{j k}(\theta)\right)$ is the differential of the spectral matrix, in the angular coordinate $\theta$, of the Jacobi matrix belonging to $2 \sum_{1}^{\infty} x_{n} x_{n+1}$ (cf. $[12$, loc. cit.]), the usual spectral parameter $\lambda$ being related to $\theta$ by $\lambda=2 \cos \theta$. Furthermore, it is to be noted that the restrictions on $c_{n}$, namely $c_{-n}=c_{n}$ and $\sum c_{n}^{2}<\infty$, used to ensure (39) are not even sufficient to imply that $T$ or $H$ be bounded. The relation (39) is to be compared with (34) wherein the heavier restriction (33) is assumed (guaranteeing, in particular, that $H$ be completely continuous).

3. In view of the discussion of $[12$, loc. cit. $]$, it is clear that $T_{n}$ is unitarily equivalent to the direct sum of $n$ copies of the matrix $T_{1}$. Consequently, the unitary equivalence relation (29) is at least suggested, but, in view of the explicit form of (29) (the polynomials $p_{n}(\lambda)$ satisfying $p_{n}(2 \cos \theta)=2 \cos n \theta$, cf. Appendix 2 above), apparently not directly implied.

4. See Appendix 1 above.

\section{REFERENCES}

1. B. Fuglede, A commmutativity theorem for normal operators, Proc. Nat. Acad. Sci. U.S.A. vol. 35 (1950) pp. 35-40.

2. P. R. Halmos, Commutators of operators, Amer. J. Math. vol. 74 (1952) pp. 237-240.

3. - Commutators of operators, II, ibid. vol. 76 (1954) pp. 191-198.

4. P. Hartman and A. Wintner, On the spectra of Toeplitz's matrices, ibid. vol. 72 (1950) pp. 359-366.

5. - The spectra of Toeplitz's matrices, ibid. vol. 76 (1954) pp. 867-882.

6. C. R. Putnam, On commutators of bounded matrices, ibid. vol. 73 (1951) pp. 127-131.

7. - On normal operators in Hilbert space, ibid. vol. 73 (1951) pp. 357-362.

8. - On commutators and Jacobi matrices, Proc. Amer. Math. Soc. (to appear).

9. M. Rosenblum, Perturbation of the continuous spectrum and unitary equivalence, Technical Report No. 12, Office of Ordnance Research, Department of Mathematics, University of California, December, 1955.

10. O. Toeplitz, Zur Theorie der quadratischen und bilinearen Formen von unendlichvielen Veränderlichen, Math. Ann. vol. 70 (1911) pp. 351-376. 1912.

11. D. Hilbert, Grundzilge einer allgemeinen theorie der linearen Integralgleichungen, Leipzig,

12. Mathematical Monographs, Northwestern University, vol. 1, 1941.

Purdue University,

LAFAYETTE, IND. 